\title{
Durfee-type bound for some non-degenerate complete intersection singularities
}

\author{
Dmitry Kerner and András Némethi
}

\begin{abstract}
The Milnor number, $\mu(X, 0)$, and the singularity genus, $p_{g}(X, 0)$ are fundamental invariants of isolated hypersurface singularities (more generally, of local complete intersections). The long standing Durfee conjecture (and its generalization) predicted the inequality $\mu(X, 0) \geq(n+1) ! p_{g}(X, 0)$, here $n=\operatorname{dim}(X, 0)$. Recently we have constructed counterexamples, proposed a corrected bound and verified it for the homogeneous complete intersections.

In the current paper we treat the case of germs with Newton-non-degenerate principal part when the Newton diagrams are "large enough", i.e. they are large multiples of some other diagrams. In the case of local complete intersections we prove the corrected inequality, while in the hypersurface case we prove an even stronger inequality.
\end{abstract}

\section{INTRODUCTION}

1.1. Let $(X, 0) \subset\left(\mathbb{C}^{n+r}, 0\right)$ be the germ of an isolated analytic complete intersection singularity of dimension $n$. The Milnor number and the singularity genus are fundamental local invariants. They can be defined as the defects of the corresponding global invariants. Indeed, by the finite determinacy, we can assume $(X, 0)$ to be an algebraic germ: let $X$ be a representative of $(X, 0)$. Take some projective compactification $X \subset \bar{X}$, assume no other singularities are added, i.e. $\bar{X} \backslash X$ is smooth. Take (one of) its resolution, $\tilde{X} \rightarrow \bar{X}$ and (one of) its smoothing $\bar{X}_{\epsilon}$. Then

$$
\mu(X, 0):=(-1)^{n}\left(\chi_{t o p}\left(\bar{X}_{\epsilon}\right)-\chi_{t o p}(\bar{X})\right), \quad p_{g}(X, 0):=(-1)^{n}\left(\chi_{a n}\left(\mathcal{O}_{\bar{X}}\right)-\chi_{a n}\left(\mathcal{O}_{\tilde{X}}\right)\right) .
$$

(Here $\chi_{t o p}$ is the topological Euler characteristic, while $\chi_{a n}(\mathcal{O})$ is the analytic Euler characteristic of the structure sheaf.) These invariants do not depend on the choice of the resolution/smoothing/compactification, they are totally determined by the local analytic geometry of the germ $(X, 0) \subset\left(\mathbb{C}^{n+r}, 0\right)$. (In fact, in the hypersurface case, $r=1, p_{g}$ is even preserved in the $\mu=$ const deformations, [AGLV, pg.115].)

The relation between the Milnor number and the singularity genus has been investigated for long time. For example, in the case of curves $p_{g}$ coincides with the classical $\delta$-invariant of the singularity. Then one has the relation $\delta=\frac{\mu+r-1}{2}$, [Buchweitz-Greuel1980], where $r$ is the number of local branches at the singular point.

In [Durfee1978] the inequality $\mu \geq 6 p_{g}$ was conjectured for surface singularities that are isolated complete intersections. In [Kerner-Némethi2011], [Kerner-Némethi2013] we disproved this initial inequality and proposed a modified inequality for isolated complete intersections $(X, 0) \subset\left(\mathbb{C}^{n+r}, 0\right)$ (of dimension $n>2$ and codimension $r$ ): $\mu \geq C_{n, r} p_{g}$. (For $n=2$ the only possible universal bound is $\mu \geq 4 p_{g}$.) We proved the new inequality for homogeneous complete intersections.

The combinatorial coefficient $C_{n, r}$ is defined by $C_{n, r}:=\frac{\left(\begin{array}{c}n+r-1 \\ n\end{array}\right)(n+r) !}{\left\{\begin{array}{c}n+r \\ r\end{array}\right\} r !}$. Here $\left\{\begin{array}{c}n+r \\ r\end{array}\right\}$ is the Stirling number of the second kind. For more details see $\S 2.1 .3$, now we only quote the basic property:

$$
(n+1) !=C_{n, 1}>C_{n, 2}>\cdots>C_{n, r}>\cdots>\lim _{r \rightarrow \infty} C_{n, r}=2^{n} .
$$

For the history and the list of other (partial) verifications see [Kerner-Némethi2013]. For the relevant notions from Singularity Theory see [AGLV], [Dimca], [Looijenga], [Oka].

This paper is the continuation of [Kerner-Némethi2011] and [Kerner-Némethi2013]. We verify the corrected bound for several additional classes of singularities.

1.2. Our first main result is the following:

Theorem 1.1. Consider an isolated complete intersection singularity, $(X, 0)=\left\{f_{1}=\cdots=f_{r}=0\right\} \subset\left(\mathbb{C}^{n+r}, 0\right)$. Suppose either $r=1, n \geq 2$, or $r>1, n>2$. Suppose the tuple $\left(f_{1}, \ldots, f_{r}\right)$ is Newton-non-degenerate with respect to the diagrams $\left(\Gamma_{1}, \ldots, \Gamma_{r}\right)$. Suppose that all the diagrams are convenient and 'large enough'. (Namely, for $i=1, \ldots, r$ : $\Gamma_{i}=d_{i} \tilde{\Gamma}_{i}$, where $1 \ll d_{i} \in \mathbb{Q}$ and $\tilde{\Gamma}_{i}$ are some other fixed Newton diagrams. $)$ Then $\mu(X, 0)>C_{n, r} p_{g}(X, 0)$.

Further, the bound is asymptotically sharp (i.e. $\frac{\mu}{p_{g}} \rightarrow C_{n, r}$ for $\max \left\{d_{i}\right\}_{i} \rightarrow \infty$ ) iff $\Gamma_{1}=\cdots=\Gamma_{r}$.

Date: February 27, 2015.

D.K. was supported by the grant FP7-People-MCA-CIG, 334347. A.N. was supported by OTKA Grant 100796. 
Remark 1.2. 1. For Newton-non-degenerate singularities the Milnor number and the singularity genus are determined combinatorially by the Newton diagrams. Therefore in this case the proof of the inequality consists of a lattice point count and its comparison to the volume(s) of the bodies under the Newton diagrams.

2. Even with this reduction to combinatorics, the proof is not straightforward. It is heavily based on an 'inequality of averages', a Fortuin-Kasteleyn-Ginibre-type result, which we prove separately in [Kerner-Némethi2014].

3. In [Kerner-Némethi2013] we have considered isolated complete intersections, when all $\left\{f_{i}\right\}$ are homogeneous. In that case we proved that the bound is asymptotically sharp precisely when all the multiplicities coincide. Therefore, our present sharpness statement ('the bound is sharp iff $\Gamma_{1}=\cdots=\Gamma_{r}$ ') is the natural extension of this fact.

4. Recall that $p_{g}$ is defined for singularities over any algebraically closed field $\mathbb{k}$ of zero characteristic. The Milnor number is a topological invariant, but in some cases it can be defined also for singularities over $\mathbb{k}$, and it satisfies the usual properties of the 'classical' Milnor number. Our proof is purely combinatorial, it does not use any complex topology. Therefore, if one defines a Milnor number over $\mathbb{k}$, with the usual properties (in particular if the results of Kouchnirenko-Khovanskii-Bivia-Ausina hold), then our proof holds over $\mathbb{k}$ as well.

1.3. For hypersurface singularities which are Newton-non-degenerate and have large enough Newton diagram, we prove in $\S 4$ a stronger inequality:

Theorem 1.3. Assume $n>2$ and let $(X, 0) \subset\left(\mathbb{C}^{n+1}, 0\right)$ be the germ of an isolated hypersurface singularity, nondegenerate with respect to its Newton diagram. Let $p=$ mult $(X, 0)$ be its multiplicity. Suppose the Newton diagram of $(X, 0)$ is 'large enough', i.e. $\Gamma_{(X, 0)}=d \tilde{\Gamma}$, where $0 \ll d \in \mathbb{Q}$, while $\tilde{\Gamma}$ is some other Newton diagram.

1. Then $\mu(X, 0)-\left((p-1)^{n+1}-\frac{p !}{(p-n-1) !}\right) \geq(n+1) ! p_{g}(X, 0)$.

2. If, moreover, the projectivized tangent cone, $\mathbb{P} T_{(X, 0)}$, has at most isolated singularities, with total Milnor number $\mu\left(\mathbb{P} T_{(X, 0)}\right)$, then

$$
\mu(X, 0)-\mu\left(\mathbb{P} T_{(X, 0)}\right)-\left((p-1)^{n+1}-\frac{p !}{(p-n-1) !}\right) \geq(n+1) ! p_{g}(X, 0) .
$$

Here the equality holds iff $\mathbb{P} T_{(X, 0)}$ is smooth, i.e. $(X, 0)$ is a homogeneous isolated hypersurface singularity.

If the projective hypersurface $\mathbb{P} T_{(X, 0)} \subset \mathbb{P}^{n}$ has only isolated singularities then the total Milnor number, $\mu\left(\mathbb{P} T_{(X, 0)}\right)$, is the sum of the local Milnor numbers, in particular it is positive. When $\mathbb{P} T_{(X, 0)}$ has non-isolated singularities the total Milnor number $\mu\left(\mathbb{P} T_{(X, 0)}\right)$, defined in equation (1), can be negative, so this term is not added in the first part.

Probably one can extend this type of stronger inequality to the complete intersections and prove:

$$
\mu-C_{n, r} p_{g}>\sum_{\underline{k} \in \mathcal{K}_{n, r}} \operatorname{Vol}_{n+r}\left(\left(\Gamma_{1}^{+}\right)^{k_{1}}, \ldots,\left(\Gamma_{r}^{+}\right)^{k_{r}}\right)\left((n+r) !-C_{n, r}\left(\begin{array}{c}
n+r \\
k_{1}, k_{2}, \ldots, k_{r}
\end{array}\right)\right) .
$$

Here the right hand side is often of the order of $\mu$. It vanishes when all the diagrams are proportional (in particular it vanishes for $r=1$ ). So, this right hand side cannot be seen when all $f_{i}$ are ordinary multiple points (i.e. isolated homogeneous singularities) or in the case of hypersurface singularities.

1.4. As one sees above, for Newton-non-degenerate singularities we always assume that the diagram(s) is/are 'large enough'. As of now we could not prove the Durfee bound for an arbitrary Newton diagram, even for Newton-nondegenerate surface singularities in $\left(\mathbb{C}^{3}, 0\right)$. In this case the combinatorial formulas are:

$$
\mu=\mu(\Gamma)=3 ! \operatorname{Vol}_{3}(\Gamma)-2 ! \operatorname{Vol}_{2}(\Gamma)+\operatorname{Vol}_{1}(\Gamma)-1, \quad p_{g}=p_{g}(\Gamma)=\left|\Gamma^{-} \cap \mathbb{Z}_{>0}^{3}\right|
$$

while the conjectural bound is $\mu(\Gamma) \geq 6 p_{g}(\Gamma)$. (Recall that $V o l_{i}$ denotes the normalized $i$-dimensional lattice volume, as e.g. in [Kouchnirenko1976].)

It is natural to try to extend this (purely combinatorial) bound to some more general class of lattice polytopes. The situation is highly delicate as the following example shows.

Example 1.4. Suppose instead of Newton diagrams in $\mathbb{R}_{\geq 0}^{3}$ one considers a generalized version: Newton diagrams inside the cone Cone $x_{1}^{-1} x_{2}^{-1} x_{3}^{-1}\left(x_{1}^{m}, x_{2}^{m}, x_{3}^{m}\right)$. (This cone is generated by the rays starting from $x_{1}^{-1} x_{2}^{-1} x_{3}^{-1}$ and passing through any of $x_{1}^{m}, x_{2}^{m}, x_{3}^{m}$.) This means that we consider Newton-non-degenerate hypersurfaces inside a toric variety with an isolated singularity. Consider the Newton diagram of the homogeneous $\Gamma=\operatorname{Conv}\left(x_{1}^{m}, x_{2}^{m}, x_{3}^{m}\right)$. Then $\Gamma^{-}=$ $\operatorname{Conv}\left(x_{1}^{-1} x_{2}^{-1} x_{3}^{-1}, x_{1}^{m}, x_{2}^{m}, x_{3}^{m}\right)$ and its parameters are (see $\left.\S 2.2\right)$ :

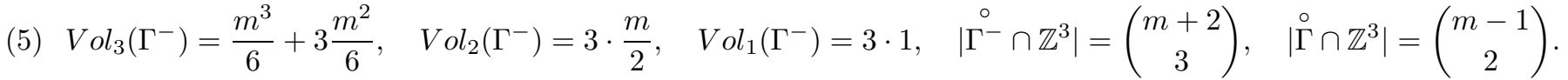


Then the singularity invariants are (see $\S 2.5)$ :

$$
\mu\left(\Gamma^{-}\right)=m^{3}+3 m^{2}-3 m+2, \quad p_{g}\left(\Gamma^{-}\right)=\left(\begin{array}{c}
m+2 \\
3
\end{array}\right)+\left(\begin{array}{c}
m-1 \\
2
\end{array}\right) .
$$

Therefore: $\mu\left(\Gamma^{-}\right)-6 p_{g}\left(\Gamma^{-}\right)=-3 m^{2}+4 m-4<0$, i.e. for this $\Gamma^{-}$the inequality is violated.

Therefore, when trying to prove the inequality in the ordinary case, $\Gamma^{-} \subset \mathbb{R}^{3}$, we cannot subdivide the body $\Gamma^{-}$into some suitable pieces and combine the total $\mu>6 p_{g}$ from its building blocks. Geometrically, this inequality cannot be proven by any local consideration of the resolution of $(X, 0)$, rather it depends on its global properties.

\section{Preliminaries}

\subsection{Some relevant combinatorics.}

2.1.1. Stirling numbers. For any $n \geq 0$ and $r \geq 1$, the Stirling number of the second kind, $\left\{\begin{array}{c}n+r \\ r\end{array}\right\}$, is the number of (unordered) partitions of $n+r$ elements into $r$ non-empty sets, see [Abramowitz-Stegun, §24.1.4, pg. 824]. We record some of its basic properties:

- $\left\{\begin{array}{l}n \\ 1\end{array}\right\}=1,\left\{\begin{array}{l}r \\ r\end{array}\right\}=1$

- for $r>1:\left\{\begin{array}{c}n+r \\ r\end{array}\right\} \geq\left\{\begin{array}{c}n+r-1 \\ r-1\end{array}\right\}$ and the equality occurs only for $n=0$;

- the generating function for these numbers is $\left(e^{x}-1\right)^{r}=r ! \sum_{n=0}^{\infty}\left\{\begin{array}{c}n+r \\ r\end{array}\right\} \frac{x^{n+r}}{(n+r) !}$;

- the explicit expansion: $\left\{\begin{array}{c}n+r \\ r\end{array}\right\}=\frac{1}{r !} \sum_{j=0}^{r}(-1)^{j}\left(\begin{array}{c}r \\ j\end{array}\right)(r-j)^{n+r}$;

- the asymptotic growth: $\left\{\begin{array}{c}n+r \\ r\end{array}\right\} \sim \frac{r^{2 n}}{2^{n} n !}$, as $r \rightarrow \infty$;

- the recurrence relation: $\left\{\begin{array}{c}n+1+r \\ r\end{array}\right\}=r\left\{\begin{array}{c}n+r \\ r\end{array}\right\}+\left\{\begin{array}{c}n+r-1 \\ r-1\end{array}\right\}$;

- for $n+r \geq r \geq j$ there is another recurrence relation: $\left(\begin{array}{l}r \\ j\end{array}\right)\left\{\begin{array}{c}n+r \\ r\end{array}\right\}=\sum_{i=r-j}^{n+r-j}\left(\begin{array}{c}n+r \\ i\end{array}\right)\left\{\begin{array}{c}n-i+j \\ j\end{array}\right\}\left\{\begin{array}{c}i+r-j \\ r-j\end{array}\right\}$.

2.1.2. The set of compositions. Denote by $\mathcal{K}_{n, r}$ the set of the (ordered) compositions,

$$
\mathcal{K}_{n, r}:=\left\{\underline{k}=\left(k_{1}, \ldots, k_{r}\right): k_{i} \geq 0 \text { for all } i \text {, and } \sum_{i} k_{i}=n\right\} .
$$

This $\mathcal{K}_{n, r}$ can be thought of as the lattice points of the simplex. Its cardinality is $\left|\mathcal{K}_{n, r}\right|=\left(\begin{array}{c}n+r-1 \\ n\end{array}\right)$.

The permutation group on $r$ elements, $\Xi_{r}$, acts on $\mathcal{K}_{n, r}$. The quotient $\mathcal{K}_{n, r} \Xi_{r}$ is the set of partitions. (Recall that a partition is an unordered composition.) For convenience we put $\mathcal{K}_{n, r}=\varnothing$ when $r \leq 0$ or $n<0$.

Suppose a set of objects is indexed by this set of compositions, $\left\{A_{\underline{k}}\right\}_{\underline{k} \in \mathcal{K}_{n, r}}$. We often use the standard set-theoretic inclusion-exclusion formula:

$$
\sum_{\underline{k} \in \mathcal{K}_{n, r}} A_{\underline{k}}-\sum_{i=1}^{r} \sum_{\substack{k \in \mathcal{K}_{n, r} \\
k_{i}=0}} A_{\underline{k}}+\sum_{\substack { 1 \leq i_{1}<i_{2} \leq r \\
\begin{subarray}{c}{\underline{k} \in \mathcal{K}_{n, r} \\
k_{i_{1}}=0=k_{i_{2}}{ 1 \leq i _ { 1 } < i _ { 2 } \leq r \\
\begin{subarray} { c } { \underline { k } \in \mathcal { K } _ { n , r } \\
k _ { i _ { 1 } } = 0 = k _ { i _ { 2 } } } }\end{subarray}} A_{\underline{k}}-\cdots=\sum_{\substack{\underline{k} \in \mathcal{K}_{n, r} \\
k_{1}, \ldots, k_{r}>0}} A_{\underline{k}} .
$$

2.1.3. The coefficient $C_{n, r}$. Using these notions the coefficient $C_{n, r}$ is defined by

$$
C_{n, r}:=\frac{\left(\begin{array}{c}
n+r-1 \\
n
\end{array}\right)(n+r) !}{\left\{\begin{array}{c}
n+r \\
r
\end{array}\right\} r !}=\frac{\left|\mathcal{K}_{n, r}\right|}{\sum_{\underline{k} \in \mathcal{K}_{n, r}} \prod_{i=1}^{r} \frac{1}{\left(k_{i}+1\right) !}} .
$$

The second equality of (9) follows from [Jordan1965, pages 176-178].

We record some properties of $C_{n, r}$.

1. $C_{n, 1}=(n+1) !, C_{n, 2}=\frac{(n+2) !(n+1)}{2^{n+2}-2}, C_{n, 3}=\frac{\left(\begin{array}{c}n+2 \\ 2\end{array}\right)(n+3) !}{3^{n+3}-3 \cdot 2^{n+3}+3}$, by direct computation.

2. $\lim _{r \rightarrow \infty} C_{n, r}=2^{n}$. The limit can be computed using the asymptotical growth of Stirling numbers, $\S 2.1 .1$. This gives: $C_{n, r} \sim 2^{n} \frac{(n+r-1) !(n+r) !}{(r-1) ! ! ! r^{2 n}}$ with limit $2^{n}$ as $r \rightarrow \infty$. 
3. $C_{n, 1}>C_{n, 2}>\cdots>C_{n, r}>\cdots>\lim _{r \rightarrow \infty} C_{n, r}$. This is proved e.g. in [Kerner-Némethi2013, Corollary 4.2].

4. $\sum_{\underline{k} \in \mathcal{K}_{n, r}}\left[(n+r) !-C_{n, r}\left(\begin{array}{c}n+r \\ k_{1}+1, \ldots, k_{r}+1\end{array}\right)\right]=0$. This follows immediately from equation (9).

2.2. Newton diagrams. Let $f\left(x_{1}, \ldots, x_{N}\right)=\sum_{I} a_{I} \underline{x}^{I}$ be a power series, with complex coefficients. Consider the support of its monomials, $\operatorname{Supp}(f):=\left\{I \in \mathbb{Z}_{\geq 0}^{N} \mid a_{I} \neq 0\right\}$. The Newton polyhedron is defined as the convex hull $\Gamma^{+}=\Gamma_{f}^{+}:=\operatorname{Conv}\left(\operatorname{Supp}(f)+\mathbb{R}_{\geq 0}^{N}\right)$. The Newton polyhedron has compact faces and unbounded faces. The Newton diagram, $\Gamma$, is the union of all the compact faces of $\Gamma^{+}$. We always assume that the diagram is 'convenient', that is, $\Gamma$ intersects all the coordinate axes (i.e. $f$ contains all the monomials $x_{1}^{m_{1}}, \ldots, x_{N}^{m_{N}}$ ).

We use the notation $\Gamma^{-}:=\left(\mathbb{R}_{>0}^{N} \backslash \Gamma^{+}\right) \cup \Gamma$ for the part not above the diagram. Denote the set of lattice points on $\Gamma$ by $\Gamma \cap \mathbb{Z}^{N}$, similarly $\Gamma^{-} \cap \mathbb{Z}^{N}$. The notation for the lattice points strictly below the diagram is $\left(\Gamma^{-} \backslash \Gamma\right) \cap \mathbb{Z}^{N}$.

Let $\operatorname{Vol}_{N}\left(\Gamma^{-}\right)$be the (lattice) volume of $\Gamma^{-}$(with the normalization that the volume of the unit cube is 1 .) More generally, for any subset $I \subseteq\{1, \ldots, N\}$ consider the corresponding coordinate plane $L_{I}=\operatorname{Span}\left(\left\{\hat{x}_{i}\right\}_{i \in I}\right)$. (Here $\hat{x}_{i}$ is the unit vector along the $i$ 'th coordinate axis.) Define $\Gamma^{I}=\Gamma \cap L_{I}$ and $\left(\Gamma^{-}\right)^{I}=\Gamma^{-} \cap L_{I}$. Accordingly, for a fixed $j$, one has the sum of volumes of intersections with all $j$-dimensional coordinate planes:

$$
\operatorname{Vol}_{j}\left(\Gamma^{-}\right):=\sum_{\substack{I \subset\{1, \ldots, N\} \\|I|=j}} \operatorname{Vol}_{j}\left(\left(\Gamma^{-}\right)^{I}\right) .
$$

In particular, $\operatorname{Vol}_{0}\left(\Gamma^{-}\right)=1$, while $\operatorname{Vol}_{1}\left(\Gamma^{-}\right)$is the total lattice length of all the segments of the type $\operatorname{Conv}\left(\overrightarrow{0}, d_{i} \hat{x}_{i}\right)$, where $\Gamma \cap \operatorname{Span}\left(\hat{x}_{i}\right)=d_{i} \hat{x}_{i}$.

The diagram $\Gamma$ consists of many faces. Each face has its (lattice) volume inside the lattice it spans. Let $V o l_{N-1} \Gamma$ be the total volume of $\Gamma$, i.e. the sum of the volumes of the top dimensional faces.

2.3. Mixed covolumes and their convexity. Given a convenient Newton polyhedron, $\Gamma^{+} \subset \mathbb{R}_{\geq 0}^{N}$, consider its covolume, $\operatorname{CoVol}\left(\Gamma^{+}\right):=\operatorname{Vol}_{N}\left(\mathbb{R}_{\geq 0}^{N} \backslash \Gamma^{+}\right)$. Given a collection of Newton polyhedra, $\left\{\Gamma_{i}^{+}\right\}_{i}$, consider their scaled Minkowski sum, $\lambda_{1} \Gamma_{1}^{+}+\cdots+\lambda_{r} \Gamma_{r}^{+}$. The covolume of this sum is a polynomial in $\left\{\lambda_{i}\right\}$, [Kaveh-Khovanskii-2013-1, $\left.\S 10\right]$ :

$$
\operatorname{CoVol}\left(\lambda_{1} \Gamma_{1}^{+}+\cdots+\lambda_{r} \Gamma_{r}^{+}\right)=\sum_{\underline{k} \in \mathcal{K}_{N, r}}\left(\begin{array}{c}
N \\
k_{1}, \ldots, k_{r}
\end{array}\right) \operatorname{coVol}\left(\left(\Gamma_{1}^{+}\right)^{k_{1}}, \ldots,\left(\Gamma_{r}^{+}\right)^{k_{r}}\right)\left(\prod_{i=1}^{r} \lambda_{i}^{k_{i}}\right)
$$

The mixed covolumes are the (positive) coefficients $\operatorname{coVol}\left(\left(\Gamma_{1}^{+}\right)^{k_{1}}, \ldots,\left(\Gamma_{r}^{+}\right)^{k_{r}}\right)$. Here $\operatorname{coVol}\left(\left(\Gamma_{1}^{+}\right)^{k_{1}}, \ldots,\left(\Gamma_{r}^{+}\right)^{k_{r}}\right)$ is a shorthand for $\operatorname{coVol}(\underbrace{\Gamma_{1}^{+}, \ldots, \Gamma_{1}^{+}}_{k_{1}}, \ldots, \underbrace{\Gamma_{r}^{+}, \ldots, \Gamma_{r}^{+}}_{k_{r}})$, for $k_{1}+\cdots+k_{r}=N$.

We use the following basic properties of the mixed covolumes:

- They are symmetric and multilinear: $\operatorname{coVol}\left(\Gamma_{11}^{+}+\Gamma_{12}^{+}, \Gamma_{2}^{+}, \ldots, \Gamma_{N}^{+}\right)=\operatorname{coVol}\left(\Gamma_{11}^{+}, \Gamma_{2}^{+}, \ldots, \Gamma_{N}^{+}\right)+\operatorname{coVol}\left(\Gamma_{12}^{+}, \Gamma_{2}^{+}, \ldots, \Gamma_{N}^{+}\right)$.

- For the diagrams $\left\{\Gamma_{i}=d_{i} \Gamma\right\}_{i=1, \ldots, r}$ one has

$$
\operatorname{coVol}_{N}\left(\sum_{i} \lambda_{i} \Gamma_{i}^{+}\right)=\operatorname{coVol}_{N}\left(\sum_{i} d_{i} \lambda_{i} \Gamma^{+}\right)=\left(\sum_{i} d_{i} \lambda_{i}\right)^{N} \operatorname{coVol}_{N}\left(\Gamma^{+}\right)=\sum_{\underline{k} \in \mathcal{K}_{n, r}}\left(\begin{array}{c}
N \\
k_{1}, \ldots, k_{r}
\end{array}\right)\left(\prod_{i=1}^{r}\left(\lambda_{i} d_{i}\right)^{k_{i}}\right) \operatorname{coVol}_{N}\left(\Gamma^{+}\right) .
$$

- Convexity property: $\operatorname{coV} \operatorname{ol}\left(\Gamma_{1}^{+}, \Gamma_{2}^{+}, \cdots, \Gamma_{N}^{+}\right)^{2} \leq \operatorname{coVol}\left(\Gamma_{1}^{+}, \Gamma_{1}^{+}, \Gamma_{3}^{+}, \cdots, \Gamma_{N}^{+}\right) \operatorname{coV} \operatorname{ol}\left(\Gamma_{2}^{+}, \Gamma_{2}^{+}, \Gamma_{3}^{+}, \cdots, \Gamma_{N}^{+}\right),[$Teissier1978], [Teissier2004, Appendix], [Kaveh-Khovanskii-2013-2, Theorem 10.5].

- In the proof of theorem 1.1 we use the following generalization of the convexity of the mixed co-volumes:

$$
\begin{aligned}
\left(\sum_{\underline{k} \in \mathcal{K}_{n, r}}\left(\begin{array}{c}
n+r \\
k_{1}+1, \ldots, k_{r}+1
\end{array}\right)\right) & \sum_{\substack{k \in \mathcal{K}_{n+r, r} \\
k_{1}, \ldots, k_{r} \geq 1}} \operatorname{coVol}_{n+r}\left(\left(\Gamma_{1}^{+}\right)^{k_{1}}, \ldots,\left(\Gamma_{r}^{+}\right)^{k_{r}}\right) \geq \\
& \geq\left(\begin{array}{c}
n+r-1 \\
n
\end{array}\right) \cdot \sum_{\substack{k \in \mathcal{K}_{n+r, r} \\
k_{1}, \ldots, k_{r} \geq 1}}\left(\begin{array}{c}
n+r \\
k_{1}+1, \ldots, k_{r}+1
\end{array}\right) \operatorname{coVol}_{n+r}\left(\left(\Gamma_{1}^{+}\right)^{k_{1}}, \ldots,\left(\Gamma_{r}^{+}\right)^{k_{r}}\right) .
\end{aligned}
$$

This inequality is proved separately in [Kerner-Némethi2014, §4]. 
2.4. Non-degeneracy with respect to Newton diagrams. The non-degeneracy notion was studied first for functions in [Kouchnirenko1976], then for complete intersections in [Khovanskii1978]). The material of this section is taken from [Bivià-Ausina2007, §3], see also [Bivià-Ausina2004].

Consider several power series, $g_{1}, \ldots, g_{r} \in \mathbb{C}\left[\left[x_{1}, \ldots, x_{N}\right]\right]$, for $r \leq N$. Take Minkowski sum of their Newton polyhedra, $\Gamma^{+}:=\Gamma_{1}^{+}+\cdots+\Gamma_{r}^{+}$. Let $\sigma$ be a compact face of $\Gamma^{+}$. By [Damon1989, Lemma 2.7] and [Bivià-Ausina2007, Lemma 3.4] there exists the unique set of compact faces, $\sigma_{1} \subset \Gamma_{1}, \ldots, \sigma_{r} \subset \Gamma_{r}$ satisfying: $\sigma=\sigma_{1}+\cdots+\sigma_{r}$.

The part of $g_{i}$ supported on $\sigma_{i}$ will be denoted by $\left.g_{i}\right|_{\sigma_{i}}$.

Definition 2.1. 1. The sequence $g_{1}, \ldots, g_{r}$ satisfies the $\left(B_{\sigma}\right)$ condition if $\left\{\left.g_{1}\right|_{\sigma_{1}}(x)=\cdots=\left.g_{r}\right|_{\sigma_{r}}(x)=0\right\} \cap\left(\mathbb{C}^{*}\right)^{N}=\varnothing$. 2. The sequence $g_{1}, \ldots, g_{r}$ is non-degenerate if it is a regular sequence (i.e. defines a subspace of codimension $r$ ) and satisfies the $\left(B_{\sigma}\right)$ condition for all the compact faces $\sigma$ of $\Gamma^{+}$of dimension $\operatorname{dim}(\sigma) \leq r-1$.

To define the non-degeneracy of the map $f=\left\{f_{1}, \ldots, f_{r}\right\}$ we need the notion of non-degeneracy of modules.

For any ideal $J$ the Newton polyhedron is defined by $\Gamma^{+}(J)=\operatorname{Conv}\left(\underset{f \in J}{\cup}\left(\operatorname{Supp}(f)+\mathbb{R}_{\geq 0}^{N}\right)\right)$. The Newton diagram (or the diagram of exponents) is defined as in $\S 2.2$.

Consider a submodule of a free module, $M \subset \mathbb{C}\left[\left[x_{1}, \ldots, x_{N}\right]\right]^{\oplus r}$. Denote by $A_{M}$ its generating matrix, i.e. a $r \times s$ matrix with entries in $\mathbb{C}\left[\left[x_{1}, \ldots, x_{N}\right]\right]$, whose columns generate the module. Denote by $M_{i}$ the ideal in $\mathbb{C}\left[\left[x_{1}, \ldots, x_{N}\right]\right]$ generated by the entries of $i$ th row of $A_{M}$. (It does not depend on the choice of generators of the module.) The Newton polyhedron of $M$ is defined to be $\Gamma^{+}(M):=\Gamma^{+}\left(M_{1}\right)+\cdots+\Gamma^{+}\left(M_{r}\right)$. (Here each $M_{i}$ is an ideal and we use the definition of $\Gamma^{+}(J)$ as above. In the case of one-row-matrix $M$ itself is an ideal.) For any compact face $\sigma$ of $\Gamma^{+}(M)$ take its (unique) presentation $\sigma=\sigma_{1}+\cdots+\sigma_{r}, \sigma_{i} \subset \Gamma^{+}\left(M_{i}\right)$, as above. Denote by $\left.M\right|_{\sigma}$ the matrix of restrictions, its $i$ 'th row consists of the restrictions onto $\sigma_{i}$. (Note that all the restrictions are polynomials, not just power series.)

Definition 2.2. The module/matrix $M$ is called Newton-non-degenerate if for any compact face $\sigma \subset \Gamma^{+}(M)$ :

$$
\left\{x \in \mathbb{C}^{N}: \operatorname{rank}\left(\left.M\right|_{\sigma}(x)\right) \leq r\right\} \cap\left(\mathbb{C}^{*}\right)^{N}=\varnothing .
$$
locus:

Finally, for a map $\underline{f}=\left(f_{1}, \ldots, f_{r}\right):\left(\mathbb{C}^{N}, 0\right) \rightarrow\left(\mathbb{C}^{r}, 0\right)$ consider a version of degeneracy matrix, describing the singular

$$
N(\underline{f}):=\left(\begin{array}{cccccc}
x_{1} \frac{\partial f_{1}}{\partial x_{1}} & \ldots & x_{n} \frac{\partial f_{1}}{\partial x_{n}} & f_{1} & \ldots & 0 \\
\ldots & \ldots & \ldots & \ldots & \ldots & \ldots \\
x_{1} \frac{\partial f_{r}}{\partial x_{1}} & \ldots & x_{n} \frac{\partial f_{r}}{\partial x_{n}} & 0 & \ldots & f_{r}
\end{array}\right) .
$$

Definition 2.3. Consider the map $\underline{f}=\left(f_{1}, \ldots, f_{r}\right):\left(\mathbb{C}^{N}, 0\right) \rightarrow\left(\mathbb{C}^{r}, 0\right)$. Suppose $f_{1}, \ldots, f_{r}$ are convenient. The map $\underline{f}$ is called Newton-non-degenerate if

(i) the sequences $f_{1}, \ldots, f_{p}$ are non-degenerate for any $p=1, \ldots, r-1$, and

(ii) the submodule $N(\underline{f}) \subset \mathbb{C}\left[\left[x_{1}, \ldots, x_{N}\right]\right]^{\oplus r}$ is Newton-non-degenerate.

Remark 2.4. In the non-hypersurface case this notion of non-degeneracy notion is more restrictive than the original definition of [Kouchnirenko1976], [Khovanskii1978]. Still, for a fixed set of diagrams it is a generic property. As we are interested only in the topological invariants, $\mu, p_{g}$, we can always assume that the complete intersection $(X, 0)$ is non-degenerate in this strict sense, and use the formulas of the next subsection.

\subsection{Invariants for Newton-non-degenerate complete intersection singularities.}

2.5.1. Milnor number for Newton-non-degenerate singularities. Let $(X, 0) \subset\left(\mathbb{C}^{N}, 0\right)$ be an isolated hypersurface singularity, non-degenerate with respect to its Newton diagram $\Gamma$. Assume that $\Gamma$ is convenient. In this case the Milnor number was computed by [Kouchnirenko1976]: $\mu(X, 0)=\sum_{0 \leq i \leq N}(-1)^{N-i} i ! \operatorname{Vol}_{i}\left(\Gamma^{-}\right)$.

The Milnor number of a Newton-non-degenerate complete intersection singularity, $(X, 0) \subset\left(\mathbb{C}^{N}, 0\right)$, was obtained in [Bivià-Ausina2007, Theorem 3.9]:

$$
\begin{aligned}
& \mu(X, 0)=\sum_{j=r}^{n+r}(-1)^{n+r-j}\left(\sum_{\substack{I \subseteq\{1, \ldots, n+r\} \\
|I|=j}} j ! a_{j}\left(\left(\Gamma_{1}^{+}\right)^{I}, \ldots,\left(\Gamma_{r}^{+}\right)^{I}\right)\right)+(-1)^{n+1}, \text { where } \\
& a_{j}\left(\left(\Gamma_{1}^{+}\right)^{I}, \ldots,\left(\Gamma_{r}^{+}\right)^{I}\right):=\sum_{\substack{\underline{k} \in \mathcal{K}_{j, r} \\
k_{1}, \ldots, k_{r} \geq 1}} \operatorname{coVol}_{j}\left(\left(\left(\Gamma_{1}^{+}\right)^{I}\right)^{k_{1}}, \ldots,\left(\left(\Gamma_{r}^{+}\right)^{I}\right)^{k_{r}}\right) .
\end{aligned}
$$

Here $I$ runs over all the coordinate planes, $\left(\Gamma_{j}^{+}\right)^{I}$ is the intersection of $\Gamma_{j}^{+}$with the $I$-th coordinate plane. The coefficient $\operatorname{coVol}_{j}\left(\left(\left(\Gamma_{1}^{+}\right)^{I}\right)^{k_{1}}, \ldots,\left(\left(\Gamma_{r}^{+}\right)^{I}\right)^{k_{r}}\right)$ is the $j$-dimensional mixed-covolume, defined in 2.3 . 
In the particular case, when all the diagrams are proportional, i.e. $\Gamma_{j}^{+}=d_{j} \Gamma^{+}, d_{j} \in \mathbb{Q}_{>0}$, one gets:

$$
\begin{aligned}
& \mu(X, 0)=\sum_{j=r}^{n+r}(-1)^{n+r-j}\left(\Theta_{j}\left(d_{1}, \ldots, d_{r}\right) j ! \operatorname{Vol}_{j}\left(\Gamma^{-}\right)\right)+(-1)^{n+1}, \quad \text { where } \\
& \Theta_{j}\left(d_{1}, \ldots, d_{r}\right):=\left(\prod_{i=1}^{r} d_{i}\right) \sum_{\underline{k} \in \mathcal{K}_{n, r}}\left(\prod_{i=1}^{r} d_{i}^{k_{i}}\right) .
\end{aligned}
$$

This was obtained in [Oka.1990, p.27], for $\left\{d_{i}\right\}$ integers and in [Bivià-Ausina2007, Corollary 6.12], for $\left\{d_{i}\right\}$ rational. The case when the Newton diagrams of $f_{1}, \ldots, f_{r}$ are 'very close' was clarified in [Martin-Pfister]. (Here 'very close' means that all $f_{i}$ are non-degenerate with respect to the 'common' diagram $\Gamma$, defined by the union of supports $\cup_{i} S u p p\left(f_{i}\right)$.)

2.5.2. Singularity genus for Newton-non-degenerate singularities. For a Newton-non-degenerate hypersurface singularity the singularity genus is expressible as $p_{g}(X, 0)=p_{g}\left(\Gamma^{+}\right):=\left|\Gamma^{-} \cap \mathbb{Z}_{>0}^{n+1}\right|$, the number of strictly positive lattice point in $\Gamma^{-}$. (This was proven for curves by Hodge (1928) and in higher dimensions in [Merle-Teissier], [Khovanskii1978], [Saito1981].)

For Newton-non-degenerate ICIS the expression for the singularity genus is the following, cf. [Khovanskii1978], [Morales1984, Theorem 2.4],

$$
p_{g}(X, 0)=p_{g}\left(\sum_{j=1}^{r} \Gamma_{j}^{+}\right)-\sum_{i=1}^{r} p_{g}\left(\sum_{j \neq i} \Gamma_{j}^{+}\right)+\sum_{1<i_{1}<i_{2} \leq r} p_{g}\left(\sum_{j \notin\left\{i_{1}, i_{2}\right\}} \Gamma_{j}^{+}\right)-\cdots+(-1)^{r+1} \sum_{j=1}^{r} p_{g}\left(\Gamma_{j}^{+}\right) .
$$

2.6. Ehrhart polynomial. Let $\Delta \subset \mathbb{Z}^{N}$ be a convex lattice polytope. Let $k \Delta \subset \mathbb{Z}^{N}$ be the polytope obtained by homogeneous $k$-scaling. The number of lattice points in $k \Delta$ can be expressed by the Ehrhart polynomial of $\Delta$ :

$$
\left|k \Delta \cap \mathbb{Z}^{N}\right|=k^{N} \operatorname{Vol}_{N}(\Delta)+\frac{k^{N-1}}{2} \operatorname{Vol}_{N-1}(\Delta)+\sum_{i=1}^{N-2} c_{i} k^{i}+1,
$$

where $\operatorname{Vol}_{N}(\Delta)$ is the lattice $N$-dimensional volume, $\operatorname{Vol}_{N-1}(\Delta)$ is the $(N-1)$-dimensional lattice volume of all the top dimensional faces of $\Delta$. The remaining coefficients $\left\{c_{1}, \ldots, c_{N-2}\right\}$ are complicated. The number of lattice points lying in the interior $k \Delta$ of $k \Delta$ is expressible as:

$$
\left|\stackrel{\circ}{\circ \Delta} \cap \mathbb{Z}^{N}\right|=k^{N} \operatorname{Vol}_{N}(\Delta)-\frac{k^{N-1}}{2} \operatorname{Vol}_{N-1}(\Delta)+\sum_{i=1}^{N-2}(-1)^{N-i} c_{i} k^{i}+(-1)^{N} .
$$

For a polygon in $\mathbb{R}^{2}$ Ehrhart formulas reduce to the classical Pick's theorem:

$$
\left|k \Delta \cap \mathbb{Z}^{N}\right|=k^{2} \operatorname{Vol}_{2}(\Delta)+\frac{k}{2} \operatorname{Vol}_{1}(\Delta)+1, \quad\left|\stackrel{\circ}{k} \Delta \cap \mathbb{Z}^{N}\right|=k^{2} \operatorname{Vol}_{2}(\Delta)-\frac{k}{2} \operatorname{Vol}_{1}(\Delta)+1 .
$$

Example 2.5. To obtain the expression for $p_{g}(\Gamma)=p_{g}\left(\Gamma^{-}\right)$, i.e. the number of $\mathbb{Z}_{>0}^{N}$ points on or under $\Gamma$, present $\Gamma^{-}=\Delta \backslash\left(\Gamma^{+} \cap \Delta\right)$. Here $\Delta$ is a large enough convex polytope that lies in $\mathbb{R}_{\geq 0}^{N}$ and contains $\Gamma^{-}$. Then: $p_{g}(\Gamma)=$

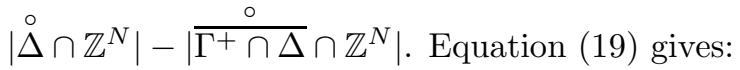

$$
p_{g}(\Gamma)=p_{g}\left(\Gamma^{-}\right)=\operatorname{Vol}_{N}\left(\Gamma^{-}\right)+\frac{\operatorname{Vol}_{N-1}(\Gamma)-V o l_{N-1}\left(\Gamma^{-}\right)}{2}+\sum_{i=1}^{N-2}(-1)^{N-i} k^{i}\left(c_{i}(\Delta)-c_{i}\left(\Gamma^{+} \cap \Delta\right)\right) .
$$

\section{Proof of the bound for large enough NeWton-NON-DEgenerate Complete intersections}

Here we prove theorem 1.1. The proof goes in 2 steps. First, we reduce the problem to a combinatorial statement, by expressing $\mu$ and $p_{g}$ in terms of the (mixed-)covolumes, $\left\{\operatorname{coVol}\left(\left(\Gamma_{1}^{+}\right)^{k_{1}}, \ldots,\left(\Gamma_{r}^{+}\right)^{k_{r}}\right)\right\}_{\left(k_{1}, \ldots, k_{r}\right)}$. Then we compare the leading terms of $\mu$ and $p_{g}$ and prove l.t. $(\mu) \geq$ l.t. $\left(p_{g}\right)$ with equality only in the case $\Gamma_{1}=\cdots=\Gamma_{r}$. This proves the theorem when not all the diagrams coincide. Finally, in the case $\Gamma_{1}=\cdots=\Gamma_{r}$, we prove the theorem by comparison of the second-order terms.

Step 1. Consider the isolated complete intersection singularity, $(X, 0)=\left\{f_{1}=\cdots=f_{r}=0\right\} \subset\left(\mathbb{C}^{n+r}, 0\right)$, Newtonnon-degenerate with respect to the diagrams $\left(\Gamma_{1}, \ldots, \Gamma_{r}\right)$. Suppose all the diagrams are convenient. The expressions for $\mu(X, 0), p_{g}(X, 0)$ are given in $\S 2.5$.

We assume all the diagrams $\Gamma_{i}$ to be large enough, in particular $\operatorname{Vol}_{n+r}\left(\Gamma_{i}^{-}\right) \gg \operatorname{Vol}_{n+r-1}\left(\Gamma_{i}^{-}\right) \gg \cdots$. Thus in the comparison of $\mu$ vs $p_{g}$ it is enough to compare only the higher order terms. First we compare the leading terms. 
The leading term for $p_{g}(\Gamma)$ is obtained from Ehrhart expansion, equation (21): l.t. $\left(p_{g}(\Gamma)\right)=\operatorname{Vol}_{n+r}\left(\Gamma^{-}\right)$. Thus equation (17) gives:

$$
\begin{aligned}
\text { l.t. }\left(p_{g}(X, 0)\right)=\operatorname{coVol}_{n+r}\left(\sum_{j=1}^{r} \Gamma_{j}^{+}\right)-\sum_{i=1}^{r} \operatorname{coVol}_{n+r}\left(\sum_{j \neq i} \Gamma_{j}^{+}\right)+ & \\
& +\sum_{1<i_{1}<i_{2} \leq r} \operatorname{coVol}_{n+r}\left(\sum_{j \notin\left\{i_{1}, i_{2}\right\}} \Gamma_{j}^{+}\right)-\cdots+(-1)^{k+1} \sum_{j=1}^{r} \operatorname{coVol}_{n+r}\left(\Gamma_{j}^{+}\right) .
\end{aligned}
$$

Expand all the brackets using mixed covolumes, $\S 2.3$, to get:

$$
\begin{aligned}
\text { l.t. }\left(p_{g}(X, 0)\right)= & \sum_{\underline{k} \in \mathcal{K}_{n+r, r}}\left(\begin{array}{c}
n+r \\
k_{1}, \ldots, k_{r}
\end{array}\right) \operatorname{coVol}_{n+r}\left(\left(\Gamma_{1}^{+}\right)^{k_{1}}, \ldots,\left(\Gamma_{r}^{+}\right)^{k_{r}}\right)- \\
& \quad-\sum_{i=1}^{r} \sum_{\substack{k \in \mathcal{K}_{n+r, r} \\
k_{i}=0}}\left(\begin{array}{c}
n+r \\
k_{1}, \ldots, k_{r}
\end{array}\right) \operatorname{coVol}_{n+r}\left(\left(\Gamma_{1}^{+}\right)^{k_{1}}, \ldots,\left(\Gamma_{r}^{+}\right)^{k_{r}}\right)+\cdots+(-1)^{k+1} \sum_{j=1}^{r} \operatorname{coVol}_{n+r}\left(\Gamma_{j}^{+}\right) .
\end{aligned}
$$

By the exclusion-inclusion formula, equation (8), we get:

$$
\text { l.t. }\left(p_{g}(X, 0)\right)=\sum_{\substack{k \in \mathcal{K}_{n+r, r} \\
k_{1}, \ldots, k_{r} \geq 1}}\left(\begin{array}{c}
n+r \\
k_{1}, \ldots, k_{r}
\end{array}\right) \operatorname{coVol}_{n+r}\left(\left(\Gamma_{1}^{+}\right)^{k_{1}}, \ldots,\left(\Gamma_{r}^{+}\right)^{k_{r}}\right) .
$$

The leading term of $\mu$ is immediate:

$$
\text { l.t. }(\mu(X, 0))=(n+r) ! \sum_{\substack{k \in \mathcal{K}_{n+r, r} \\ k_{1}, \ldots, k_{r} \geq 1}} \operatorname{coVol}\left(\left(\Gamma_{1}^{I}\right)^{k_{1}}, \ldots,\left(\Gamma_{r}^{I}\right)^{k_{r}}\right) .
$$

To prove the initial equality it is enough to check l.t. $(\mu(X, 0))>C_{n, r} p_{g}(X, 0)$. We prove:

$$
\text { l.t. }(\mu(X, 0)) \geq C_{n, r} p_{g}(X, 0) \text {, and equality occurs iff } \Gamma_{1}=\cdots=\Gamma_{r} .
$$

(For example, the equality occurs in the hypersurface case, $r=1$.) But this is exactly the inequality presented in equation (13), proved in [Kerner-Némethi2014, §4].

Step 2. The comparison of the leading terms, as above, proves $\mu>C_{n, r} p_{g}$ when at least two diagrams among $\left\{\Gamma_{i}\right\}$ do not coincide. It remains to check the case $\Gamma_{1}=\cdots=\Gamma_{r}$. In this case the expressions for $\mu$ and $p_{g}$ simplify:

$$
\begin{aligned}
& \mu(X, 0)=\sum_{j=r}^{n+r}(-1)^{n+r-j}\left|\mathcal{K}_{j-r, r}\right| j ! \operatorname{Vol}_{j}\left(\Gamma^{-}\right)+(-1)^{n+1}, \\
& p_{g}(X, 0)=p_{g}\left(r \Gamma^{+}\right)-r p_{g}\left((r-1) \Gamma^{+}\right)+\left(\begin{array}{l}
r \\
2
\end{array}\right) p_{g}\left((r-2) \Gamma^{+}\right)-\cdots+(-1)^{r+1} r p_{g}\left(\Gamma^{+}\right)
\end{aligned}
$$

Now the expansions by the orders of $\Gamma$ are:

$$
\begin{aligned}
& \mu(X, 0)=(n+r) !\left|\mathcal{K}_{n, r}\right| \operatorname{Vol}_{n+r}\left(\Gamma^{-}\right)-(n+r-1) !\left|\mathcal{K}_{n-1, r}\right| \operatorname{Vol}_{n+r-1}\left(\Gamma^{-}\right)+\cdots, \\
& p_{g}(X, 0)=\sum_{j=0}^{r}(-1)^{j}\left(\begin{array}{c}
r \\
j
\end{array}\right)\left(\operatorname{Vol}_{n+r}\left((r-j) \Gamma^{-}\right)+\frac{V o l_{n+r-1}((r-j) \Gamma)-V o l_{n+r-1}\left((r-j) \Gamma^{-}\right)}{2}\right)+\cdots
\end{aligned}
$$

Note that $\operatorname{Vol}_{i}\left((r-j) \Gamma^{-}\right)=(r-j)^{i} \operatorname{Vol}_{i}\left(\Gamma^{-}\right)$and $\operatorname{Vol}_{n+r-1}((r-j) \Gamma)=(r-j)^{n+r-1} \operatorname{Vol}_{n+r-1}(\Gamma)$. Thus one has:

$$
p_{g}(X, 0)=\left\{\begin{array}{c}
n+r \\
r
\end{array}\right\} V o l_{n+r}\left(\Gamma^{-}\right)+\left\{\begin{array}{c}
n+r-1 \\
r
\end{array}\right\} \frac{V o l_{n+r-1}(\Gamma)-V o l_{n+r-1}\left(\Gamma^{-}\right)}{2}+\cdots
$$

Thus we need to prove:

$$
\begin{aligned}
(n+r) !\left|\mathcal{K}_{n, r}\right| \operatorname{Vol}_{n+r}\left(\Gamma^{-}\right)-(n+r & -1) !\left|\mathcal{K}_{n-1, r}\right| \operatorname{Vol}_{n+r-1}\left(\Gamma^{-}\right)> \\
& >C_{n, r}\left(\left\{\begin{array}{c}
n+r \\
r
\end{array}\right\} \operatorname{Vol}_{n+r}\left(\Gamma^{-}\right)+\left\{\begin{array}{c}
n+r-1 \\
r
\end{array}\right\} \frac{\operatorname{Vol}_{n+r-1}(\Gamma)-\operatorname{Vol}_{n+r-1}\left(\Gamma^{-}\right)}{2}\right) .
\end{aligned}
$$


The leading terms here cancel. (This was shown in Step 1 and can be also checked explicitly: $(n+r) !\left|\mathcal{K}_{n, r}\right| V o l_{n+r}(\Gamma)=$ $\left.C_{n, r}\left\{\begin{array}{c}n+r \\ r\end{array}\right\} V o l_{n+r}\left(\Gamma^{-}\right).\right)$Therefore it remains to prove:

$$
-(n+r-1) !\left|\mathcal{K}_{n-1, r}\right| \operatorname{Vol}_{n+r-1}\left(\Gamma^{-}\right)>C_{n, r}\left\{\begin{array}{c}
n+r-1 \\
r
\end{array}\right\} \frac{\operatorname{Vol}_{n+r-1}(\Gamma)-V o l_{n+r-1}\left(\Gamma^{-}\right)}{2} .
$$

Use the definition of $C_{n, r}$ to present this in the form:

$$
\frac{V o l_{n+r-1}\left(\Gamma^{-}\right)-V o l_{n+r-1}(\Gamma)}{2} \frac{(n+r)(n+r-1)}{n} \frac{\left\{\begin{array}{c}
n+r-1 \\
r
\end{array}\right\}}{\left\{\begin{array}{c}
n+r \\
r
\end{array}\right\}}>V o l_{n+r-1}\left(\Gamma^{-}\right) .
$$

We claim that $\operatorname{Vol}_{n+r-1}(\Gamma) \leq \frac{\operatorname{Vol}_{n+r-1}\left(\Gamma^{-}\right)}{(n+r)}$. This can be seen, e.g. by the projection of $\Gamma$ on all the coordinate hyperplanes, $\left\{x_{i}=0\right\}_{i=1}^{n+r}$. Substitute this inequality and cancel $V o l_{n+r-1}(\ldots)$ It remains to prove:

$$
\left\{\begin{array}{c}
n+r-1 \\
r
\end{array}\right\} /\left\{\begin{array}{c}
n+r \\
r
\end{array}\right\}>\frac{2 n}{(n+r-1)^{2}} .
$$

For $r=1, n>2$ this inequality is verified directly: $1>\frac{2}{n}$. Thus we assume $r>1$ and use the recurrence relations of $\S 2.1 .1$. This gives:

$$
\frac{\left\{\begin{array}{c}
n+r-1 \\
r
\end{array}\right\}}{\left\{\begin{array}{c}
n+r \\
r
\end{array}\right\}}=\frac{1}{r+\frac{\left\{\begin{array}{c}
n+r-2 \\
r-1
\end{array}\right\}}{\left\{\begin{array}{c}
n+r-1 \\
r
\end{array}\right\}}}>\frac{1}{r+1}
$$

(For the later inequality see $\S 2.1 .1$.$) Therefore, it is enough to check: \frac{1}{r+1}>\frac{2 n}{(n+r-1)^{2}}$. Note that $\frac{1}{r+1}-\frac{2 n}{(n+r-1)^{2}}=$ $\frac{(r-1)^{2}+\left(n^{2}-4 n\right)}{(r+1)(n+r-1)^{2}}$. This leaves only one case to check separately: $(r, n)=(2,3)$. In this case:

$$
\frac{\left\{\begin{array}{c}
n+r-1 \\
r
\end{array}\right\}}{\left\{\begin{array}{c}
n+r \\
r
\end{array}\right\}}=\frac{\left\{\begin{array}{c}
3+2-1 \\
2
\end{array}\right\}}{\left\{\begin{array}{c}
3+2 \\
2
\end{array}\right\}}=\frac{7}{15}>\frac{6}{16}=\frac{2 \cdot 3}{(3+2-1)^{2}}=\frac{2 n}{(n+r-1)^{2}} .
$$

\section{A STRONGER ASYMPTOTIC BOUND FOR HYPERSURFACES}

The proof of theorem 1.3 is in $\S 4.2$. Although the germ $(X, 0) \subset\left(\mathbb{C}^{n+1}, 0\right)$ is a local object, the statement of the theorem contains the projective hypersurface $\mathbb{P} T_{(X, 0)} \subset \mathbb{P}^{n}$. In $\S 4.1$ we derive some facts about the Milnor number $\mu\left(\mathbb{P} T_{(X, 0)}\right)$.

4.1. An auxiliary Khovanskii-Kouchnirenko type formula. Let $\Delta \subset \mathbb{R}_{\geq 0}^{n+1}$ be a convex lattice polytope such that $\operatorname{Span}_{\mathbb{R}}(\Delta)=\mathbb{R}^{n+1}$. Let $\left(\mathbb{C}^{*}\right)^{n+1} \subset Y_{\Delta}$ be the corresponding toric completion, with the natural sheaf $\mathcal{O}_{Y_{\Delta}}(1)$. Let $D_{\infty}:=Y_{\Delta} \backslash\left(\mathbb{C}^{*}\right)^{n+1}$ be the divisor at infinity. The variety $Y_{\Delta}$ is in general non-smooth. Suppose it is smoothable, i.e. there exists a flat family $\left(\mathcal{Y}, \mathcal{L}_{\mathcal{Y}}\right)$ over $\left(\mathbb{C}^{1}, 0\right)$ such that $\left.\mathcal{Y}\right|_{0}=Y_{\Delta},\left.\mathcal{Y}\right|_{t \neq 0}$ is smooth and $\left.\mathcal{L}\right|_{\pi^{-1}(0)}=\mathcal{O}_{Y_{\Delta}}(1)$.

Let $\Delta_{0} \subset \Delta$ be a lattice sub-polytope, let $f$ be a function supported on $\Delta_{0}$ and non-degenerate with respect to $\Delta_{0}$. Let $X_{\Delta_{0}}=\overline{\{f=0\}} \subset Y_{\Delta}$ be the corresponding projective hypersurface. By construction all its singularities lie on the boundary $D_{\infty}$. Note that $X_{\Delta_{0}}$ can have non-isolated singularities.

Suppose $\operatorname{dim}\left(\Delta_{0}\right)=n+1$, in particular $\operatorname{Span}_{\mathbb{R}}\left(\Delta_{0}\right)=\mathbb{R}^{n+1}$. Let $X_{\Delta}$ be a generic (partial) smoothing of $X_{\Delta_{0}}$ inside $Y_{\Delta}$. Namely, $X_{\Delta} \subset Y_{\Delta}$ is a hypersurface, defined by $\left\{f_{t}=0\right\}$, where $\operatorname{Supp}\left(f_{t}\right)=\Delta$ and $f_{t}$ is non-degenerate on $\Delta$. So $X_{\Delta} \cap\left(\mathbb{C}^{*}\right)^{n+1}$ is smooth and $X_{\Delta}$ intersects $D_{\infty}$ transversally. Note that $X_{\Delta}$ itself is smoothable, in the family $\left(\mathcal{Y}, \mathcal{L}_{\mathcal{Y}}\right)$, and its smoothing is also a smoothing of $X_{\Delta_{0}}$.

Define the Milnor number, $\mu\left(X_{\Delta_{0}}\right):=(-1)^{n}\left(\chi\left(X_{\Delta}\right)-\chi\left(X_{\Delta_{0}}\right)\right)$.

If $Y_{\Delta}$ is itself smooth then $X_{\Delta}$ is smooth and this definition coincide with that of equation (1).

Lemma 4.1. Under the assumptions as above: $\mu\left(X_{\Delta_{0}}\right)=(n+1) ! \operatorname{Vol}_{n+1}\left(\Delta \backslash \Delta_{0}\right)-\mu\left(X_{\Delta_{0}} \cap D_{\infty}\right)$. 
Proof. By [Khovanskii1978, pg. 59]

$$
\chi\left(X_{\Delta} \cap\left(\mathbb{C}^{*}\right)^{n+1}\right)=(-1)^{n}(n+1) ! \operatorname{Vol}_{n+1}(\Delta)
$$

and similarly for $X_{\Delta_{0}}$. (Here we use the assumption that $\operatorname{dim}\left(\Delta_{0}\right)=n+1$.) Hence

$$
\mu\left(X_{\Delta_{0}}\right)=(n+1) ! \operatorname{Vol}_{n+1}\left(\Delta \backslash \Delta_{0}\right)+(-1)^{n}\left(\chi\left(X_{\Delta} \cap D_{\infty}\right)-\chi\left(X_{\Delta_{0}} \cap D_{\infty}\right)\right)
$$

Finally, as $X_{\Delta}$ intersects $D_{\infty}$ transversally and $X_{\Delta} \cap\left(\mathbb{C}^{*}\right)^{n+1}$ is smooth we obtain that if $X_{\epsilon}$ is a smoothing of $X_{\Delta_{0}}$ then $\chi\left(X_{\epsilon} \cap\left(\mathbb{C}^{*}\right)^{n+1}\right)=\chi\left(X_{\Delta} \cap\left(\mathbb{C}^{*}\right)^{n+1}\right)$, and $X_{\epsilon} \backslash\left(X_{\epsilon} \cap\left(\mathbb{C}^{*}\right)^{n+1}\right)$ is a smoothing of $X_{\Delta} \cap D_{\infty}$. Thus $\mu\left(X_{\Delta}\right)=$ $(-1)^{n}\left(\chi\left(X_{\epsilon} \backslash\left(X_{\epsilon} \cap\left(\mathbb{C}^{*}\right)^{n+1}\right)-\chi\left(X_{\Delta} \cap D_{\infty}\right)\right)\right.$, and $\mu\left(X_{\Delta_{0}}\right)=(-1)^{n}\left(\chi\left(X_{\epsilon} \backslash\left(X_{\epsilon} \cap\left(\mathbb{C}^{*}\right)^{n+1}\right)-\chi\left(X_{\Delta_{0}} \cap D_{\infty}\right)\right)\right.$.

Example 4.2. In the simplest case, suppose $\Delta=\operatorname{Conv}\left(x_{0}^{p}, \ldots, x_{n+1}^{p}\right) \subset \mathbb{R}^{n+2}$, so that $\left(Y_{\Delta}, \mathcal{O}_{Y_{\Delta}}(1) \approx\left(\mathbb{P}^{n+1}, \mathcal{O}_{\mathbb{P}^{n+1}}(p)\right)\right.$. Suppose $\Delta_{0}$ intersects all the (one-dimensional) edges of $\Delta$, then $X_{\Delta_{0}}$ has only isolated singularities. Then iterating the formula of the lemma we get Kouchnirenko's formula:

$$
\mu\left(X_{\Delta_{0}}\right)=(n+1) ! \operatorname{Vol}_{n+1}\left(\Delta \backslash \Delta_{0}\right)-(n) ! \operatorname{Vol}_{n}\left(\Delta \backslash \Delta_{0}\right)+\ldots
$$

This formula will be used in equation (41).

4.2. Proof of theorem 1.3. Let $(X, 0) \subset\left(\mathbb{C}^{n+1}, 0\right)$ be an isolated hypersurface singularity, non-degenerate with respect to its diagram $\Gamma_{(X, 0)}$.

By direct check, if $(X, 0)$ is a homogeneous isolated hypersurface singularity (and thus $\left.\mu\left(\mathbb{P} T_{(X, 0)}\right)=0\right)$, we have the equality:

$$
\mu(X, 0)-\left((p-1)^{n+1}-\frac{p !}{(p-n-1) !}\right)=(n+1) ! p_{g}(X, 0) .
$$

Therefore we assume that $(X, 0)$ is not an ordinary multiple point, in particular $\mathbb{P} T_{(X, 0)}$ is not smooth.

The combinatorial formulas for Milnor number and geometric genus of a Newton-non-degenerate singularity are given in $\S 2.5$. We want to prove: for any Newton diagram $\Gamma$ there exists $k_{0}$ such that for $k \geq k_{0}$ one has

$$
\mu(k \Gamma)-\mu\left(\mathbb{P} T_{(k X, 0)}\right)-\left((k p-1)^{n+1}-\frac{(k p) !}{(k p-n-1) !}\right)>(n+1) ! p_{g}\left(k \Gamma^{-}\right) .
$$

(Here $k X$ denotes the corresponding projective hypersurface. If the singularities of $\mathbb{P} T_{(k X, 0)}$ are non-isolated then the term $\mu\left(\mathbb{P} T_{(k X, 0)}\right)$ is omitted.) As in the proof of theorem 1.1 we expand the whole expression in powers of $k$ and prove that the leading term is positive.

Step 1. Equation (21) gives:

$$
p_{g}\left(k \Gamma^{-}\right)=k^{n+1} \operatorname{Vol}_{n+1}\left(\Gamma^{-}\right)+\frac{k^{n}}{2}\left(\operatorname{Vol}_{n} \Gamma-\operatorname{Vol}_{n} \Gamma^{-}\right)+\text {l.o.t. }
$$

The Kouchnirenko formula for Milnor number gives:

$$
\mu(k \Gamma)=k^{n+1}(n+1) ! \operatorname{Vol}_{n+1}\left(\Gamma^{-}\right)-k^{n} n ! V o l_{n}\left(\Gamma^{-}\right)+\text {l.o.t. }
$$

If the singularities of $\mathbb{P} T_{(X, 0)}$ are isolated then in particular $\operatorname{dim}\left(\Delta_{0}\right)=n$. Then lemma 4.1 reads:

$$
\mu\left(\mathbb{P} T_{(k X, 0)}\right)=n ! \operatorname{Vol}_{n}\left(k \Delta \backslash \Delta_{0}\right)-\mu\left(X_{k \Delta_{0}} \cap D_{\infty}\right) \quad \text { and the } k \text {-order of } \mu\left(X_{k \Delta_{0}} \cap D_{\infty}\right) \text { is lower than } n \text {. }
$$

Here $\Delta=\operatorname{Conv}\left(x_{1}^{p}, \ldots, x_{n+1}^{p}\right)$, while $\Delta_{0}$ is the Newton polyhedron of $\mathbb{P} T_{(X, 0)}$. In what follows we denote $\Delta_{0}$ by $\Gamma\left(\mathbb{P} T_{(X, 0)}\right)$.

Finally, expand

$$
(k p-1)^{n+1}-\frac{(k p) !}{(k p-n-1) !}=\frac{(n+1)(n-2)}{2} p^{n} k^{n}-\left(\begin{array}{c}
n+2 \\
3
\end{array}\right) \frac{3 n-7}{4} p^{n-1} k^{n-1}+\text { l.o.t. }
$$

Substitute all the data into the inequality (38) to get the expansion:

$$
\frac{k^{n}(n+1) !}{2}\left(\frac{n-1}{n+1} \operatorname{Vol}_{n} \Gamma^{-}-\operatorname{Vol}_{n} \Gamma-\frac{n-2}{n !} p^{n}-\frac{2}{n+1} \operatorname{Vol}_{n}\left(\Delta \backslash \Gamma\left(\mathbb{P} T_{(X, 0)}\right)\right)\right)+\text { l.o.t. }
$$

To prove that this expression is positive/non-negative we check the coefficient of $k^{n}$. If the singularities of $\mathbb{P} T_{(X, 0)}$ are non-isolated then we can omit the term $\Delta \backslash \Gamma\left(\mathbb{P} T_{(X, 0)}\right)$. However we prove the non-negativity even with that term. (Note that $\operatorname{Vol}_{n}\left(\Delta \backslash \Gamma\left(\mathbb{P} T_{(X, 0)}\right)\right)$ is non-negative.) 
Since $\frac{p^{n}}{n !}$ is the volume $\operatorname{Vol}_{n} \Delta$, we need to prove

$$
\frac{n-1}{n+1} \operatorname{Vol}_{n} \Gamma^{-}-\operatorname{Vol}_{n} \Gamma+\frac{2}{n+1} \operatorname{Vol}_{n} \Gamma\left(\mathbb{P} T_{(X, 0)}\right)-\left(n-2+\frac{2}{n+1}\right) \operatorname{Vol}_{n} \Delta>0
$$

Step 2. Let $\Gamma=\cup_{\alpha} \sigma_{\alpha}$ be the decomposition into the top-dimensional faces. Here $\alpha$ belongs to some set and we fix a special value $\alpha=p$ by $\sigma_{p}:=\Gamma \cap \Delta$. If $\sigma_{p}$ is not top-dimensional, then it is omitted.

Let $\pi_{j}: \mathbb{R}^{n+1} \rightarrow\left\{x_{j}=0\right\} \subset \mathbb{R}^{n+1}$ be the projection onto a coordinate hyperplane. Note that $\pi_{j}$ sends $\mathbb{Z}^{n+1}$ to $\mathbb{Z}^{n}$, in particular $\pi_{j}\left(\sigma_{\alpha}\right)$ is a lattice polytope. Consider the union of the images of such projections, $\pi \sigma_{\alpha}=\cup_{j} \pi_{j}\left(\sigma_{\alpha}\right)$.

Now, we return to inequality (44). We have

$$
V o l_{n} \Gamma=\sum_{\alpha \neq p} V o l_{n} \sigma_{\alpha}+\operatorname{Vol}_{n} \sigma_{p}, \quad V o l_{n} \Gamma^{-}=\sum_{\alpha \neq p} V o l_{n} \pi \sigma_{\alpha}+V o l_{n} \pi \sigma_{p}
$$

Here the sums $\sum_{\alpha \neq p}(\ldots)$ are non-empty as $(X, 0)$ is not an ordinary multiple point.

Note that $V o l_{n} \pi \sigma_{p}=(n+1) \operatorname{Vol}_{n} \sigma_{p}$ and $\Gamma\left(\mathbb{P} T_{(X, 0)}\right)=\sigma_{p}$. Thus the inequality (to be proved) becomes:

$$
\sum_{\alpha \neq p}\left(\frac{n-1}{n+1} V o l_{n} \pi \sigma_{\alpha}-\operatorname{Vol}_{n} \sigma_{\alpha}\right)-\left(n-2+\frac{2}{n+1}\right) V o l_{n}\left(\Delta \backslash \sigma_{p}\right)>0 .
$$

Step 3. Consider the projection $\Gamma \stackrel{\nu}{\rightarrow} \Delta$ defined by $p t \rightarrow \Delta \cap \operatorname{line}(0, p t)$. This projection is surjective as a map of points of $\Gamma$ with real coordinates. In general the lattice points of $\Gamma$ are not sent to the lattice points of $\Delta$.

The image of a face, $\nu\left(\sigma_{\alpha}\right) \subset \Delta$ is a rational polytope. Let $V_{o l} l_{n}^{\mathbb{R}}\left(\nu\left(\sigma_{\alpha}\right)\right)$ denote its rational normalized volume, namely: $\operatorname{Vol}_{n}^{\mathbb{R}}\left(\nu\left(\sigma_{\alpha}\right)\right):=\operatorname{Vol}_{n}^{\mathbb{R}}\left(\pi_{j} \nu\left(\sigma_{\alpha}\right)\right)$, for any $j$. Here $\operatorname{Vol}_{n}^{\mathbb{R}}\left(\pi_{j} \nu\left(\sigma_{\alpha}\right)\right)$ is the usual volume in the hyperplane $\mathbb{R}^{n-1}$. Note that $\operatorname{Vol}_{n}\left(\Delta \backslash \sigma_{p}\right)=\sum_{\alpha \neq p} V o l_{n}^{\mathbb{R}}\left(\nu\left(\sigma_{\alpha}\right)\right)$. Thus the inequality can be written in the form

$$
\sum_{\alpha \neq p}\left(\frac{n-1}{n+1} \operatorname{Vol}_{n} \pi \sigma_{\alpha}-\operatorname{Vol}_{n} \sigma_{\alpha}-\left(n-2+\frac{2}{n+1}\right) V o l_{n}^{\mathbb{R}}\left(\nu\left(\sigma_{\alpha}\right)\right)\right)>0 .
$$

We prove that each summand is positive.

Step 4. Suppose that the top dimensional face $\sigma_{\alpha}$ lies in the hyperplane $\sum_{j=1}^{n+1} \frac{x_{j}}{a_{j}}=$ const. Here $\left\{a_{j}\right\}$ are natural numbers and $\operatorname{gcd}\left(a_{1}, \ldots, a_{n+1}\right)=1$. Then the primitive normal to the face has coordinates: $\mathcal{N}_{\alpha}=\left(\frac{\prod a_{i}}{a_{1} d}, \ldots, \frac{\prod a_{i}}{a_{n+1} d}\right)$, where $d:=\operatorname{gcd}\left(\frac{\prod a_{i}}{a_{1}}, \ldots, \frac{\prod a_{i}}{a_{n+1}}\right)$.

Note that $\frac{V o l_{n} \pi_{j} \sigma_{\alpha}}{V o l_{n} \sigma_{\alpha}}=\frac{\prod a_{i}}{a_{j} d}$. This can be obtained by comparing the lattice areas of the $\operatorname{simplex} C o n v\left(x_{1}^{a_{1}}, \ldots, x_{n+1}^{a_{n+1}}\right)$ and its projections. Therefore

$$
\frac{n-1}{n+1} \operatorname{Vol}_{n} \pi \sigma_{\alpha}-\operatorname{Vol}_{n} \sigma_{\alpha}=\operatorname{Vol}_{n} \sigma_{\alpha}\left(\frac{n-1}{n+1} \sum_{j} \frac{\prod a_{i}}{a_{j} d}-1\right) .
$$

Now compare $V o l_{n} \sigma_{\alpha}$ to $V o l_{n}^{\mathbb{R}} \nu \sigma_{\alpha}$. We claim $V o l_{n}^{\mathbb{R}} \nu \sigma_{\alpha}<\min _{j} V o l_{n} \pi_{j} \sigma_{\alpha}$ (note that the inequality is strict). Indeed, the left hand side was defined (in Step 3.) as the real area $V o l_{n-1}^{\mathbb{R}} \pi_{j} \nu \sigma_{\alpha}$. But $V o l_{n-1}^{\mathbb{R}} \pi_{j} \nu \sigma_{\alpha}<V o l_{n} \pi_{j} \sigma_{\alpha}$.

Thus $V o l_{n-1}^{\mathbb{R}} \nu \sigma_{\alpha}<\left(\min _{j} \frac{\prod a_{i}}{a_{j} d}\right) V o l_{n} \sigma_{\alpha}$. Therefore it is enough to prove the following arithmetic statement, for $\left(a_{1}, \ldots, a_{n+1}\right) \neq(1, \ldots, 1)$ :

$$
\frac{n-1}{n+1} \sum_{j} \frac{\prod a_{i}}{a_{j} d}-1-\left(n-2+\frac{2}{n+1}\right) \min _{j} \frac{\prod a_{i}}{a_{j} d} \geq 0
$$

Note that now the inequality to be proved is non-strict. Present it in the form: $\frac{n-1}{n+1}\left(\sum_{j=1}^{n+1} \frac{\prod a_{i}}{a_{j} d}-n \cdot \min _{j} \frac{\prod a_{i}}{a_{j} d}\right) \geq 1$.

As $\mathcal{N} \neq(1, \ldots, 1)$ we have: $\sum_{j=1}^{n+1} \frac{\prod a_{i}}{a_{j} d} \geq(n+1) \cdot \min _{j} \frac{\prod a_{i}}{a_{j} d}+1$. So, the inequality becomes: $\frac{n-1}{n+1}\left(1+\min _{j} \frac{\prod a_{i}}{a_{j} d}\right) \geq 1$, which is obvious for $n \geq 3$. (Just note: $1+\min _{j} \frac{\prod a_{i}}{a_{j} d} \geq 2$ and $\frac{n-1}{n+1} \cdot 2 \geq 1$.) 


\section{REFERENCES}

[Abramowitz-Stegun] M. Abramowitz, I.A. Stegun, Handbook of mathematical functions with formulas, graphs, and mathematical tables. National Bureau of Standards Applied Mathematics Series, 55, 1964.

[AGLV] V.I. Arnol'd, V.V. Goryunov, O.V. Lyashko, V.A. Vasil'ev, Singularity theory.I. Reprint of the original English edition from the series Encyclopaedia of Mathematical Sciences [Dynamical systems. VI, Encyclopaedia Math. Sci., 6, Springer, Berlin, 1993]. Springer-Verlag, Berlin, 1998.

[Artin1966] M. Artin, On isolated rational singularities of surfaces. Amer. J. Math. 881966 129-136.

[Ashikaga1992] T. Ashikaga, Normal two-dimensional hypersurface triple points and the Horikawa type resolution. Tohoku Math. J. (2) 44 (1992), no. 2, 177-200.

[Berline-Vergne-2007] N. Berline, M. Vergne, Local Euler-Maclaurin formula for polytopes. Mosc. Math. J. 7 (2007), no. 3, 355-386, 573

[Bivià-Ausina2004] C. Bivià-Ausina, The integral closure of modules, Buchsbaum-Rim multiplicities and Newton polyhedra. J. London Math. Soc. (2) 69 (2004), no. 2, 407-427

[Bivià-Ausina2007] C. Bivià-Ausina, Mixed Newton numbers and isolated complete intersection singularities. Proc. Lond. Math. Soc. (3) 94 (2007), no. 3, 749-771.

[Buchweitz-Greuel1980] R.-O. Buchweitz, G.-M. Greuel, The Milnor number and deformations of complex curve singularities. Invent. Math. 58 (1980), no. 3, 241-281.

[Dimca] A. Dimca, Singularities and topology of hypersurfaces. Universitext. Springer-Verlag, New York, 1992.

[Damon1989] J. Damon, Topological invariants of $\mu$-constant deformations of complete intersection singularities. Quart. J. Math. Oxford Ser. (2) 40 (1989), no. 158, 139-159

[Durfee1978] A.H. Durfee, The signature of smoothings of complex surface singularities. Math. Ann. 232 (1978), no. 1, 85-98.

[Greuel1975] G.M. Greuel, Der Gauss-Manin-Zusammenhang isolierter Singularitäten von vollständigen Durchschnitten. Math. Ann. 214 (1975), 235-266.

[Greuel-Hamm1978] G.M. Greuel, H.A. Hamm, Invarianten quasihomogener vollständiger Durchschnitte., Invent. Math. 49 (1978), no. 1, $67-86$.

[Hamm1986] H.A. Hamm, Invariants of weighted homogeneous singularities. Journées Complexes 85 (Nancy, 1985), 613, Inst. Élie Cartan, 10, Univ. Nancy, Nancy, 1986.

[Hamm2011] H.A. Hamm, Differential forms and Hodge numbers for toric complete intersections, arXiv:1106.1826.

[Jordan1965] Ch. Jordan, Calculus of finite differences. Third Edition. Introduction by Harry C. Carver Chelsea Publishing Co., New York $1965 \mathrm{xxi}+655 \mathrm{pp}$

[Kantor-Khovanskii-1993] J.M. Kantor, A. Khovanskii, Une application du théorème de Riemann-Roch combinatoire au polynôme d'Ehrhart des polytopes entiers de $\mathbb{R}^{d}$ C. R. Acad. Sci. Paris Sr. I Math. 317 (1993), no. 5, 501-507

[Kaveh-Khovanskii-2013-1] K. Kaveh, A.G. Khovanskii, Convex bodies and multiplicities of ideals, arXiv:1302.2676.

[Kaveh-Khovanskii-2013-2] K. Kaveh, A.G. Khovanskii, On mixed multiplicities of ideals, arXiv:1310.7979.

[Kerner-Némethi2009] D. Kerner and A. Némethi, The Milnor fibre signature is not semi-continuous, Proc. of the Conference "Topology of Algebraic Varieties", Jaca (Spain), Contemporary Math. 538 (2011), 369-376.

[Kerner-Némethi2011] D. Kerner, A. Némethi, A counterexample to Durfee conjecture, Comptes Rendus Mathématiques de l'Académie des Sciences, vol.34 (2012), no.2. arXiv:1109.4869

[Kerner-Némethi2013] D. Kerner, A. Némethi, The 'corrected Durfee's inequality' for homogeneous complete intersections, Mathematische Zeitschrift, Math. Z. 274 (2013), no. 3-4, pp.1385-1400

[Kerner-Némethi2014] D. Kerner, A. Némethi, A generalized FKG-inequality for compositions, arXiv: 1412.8200.

[Khovanskii1978] A.G. Khovanskii, Newton polyhedra, and the genus of complete intersections. (Russian) Funktsional. Anal. i Prilozhen. 12 (1978), no. 1, 51-61.

[Kouchnirenko1976] A.G. Kouchnirenko, Polyédres de Newton et nombres de Milnor. Invent. Math. 32 (1976), no. 1, 1-31

[Laufer1977] H.B. Laufer, On $\mu$ for surface singularities, Proceedings of Symposia in Pure Math. 30, 45-49, 1977.

[Looijenga] E. Looijenga, Isolated Singular Points on Complete Intersections. London Math. Soc. LNS 77, CUP, 1984.

[Looijenga1986] E. Looijenga, Riemann-Roch and smoothings of singularities. Topology 25 (1986), no. 3, $293-302$.

[Martin-Pfister] B. Martin, G. Pfister, Milnor number of complete intersections and Newton polygons. Math. Nachr. 110 (1983), 159-177

[Melle-Hernández2000] A. Melle-Hernández, Milnor numbers for surface singularities. Israel J. Math. 115 (2000), 29-50.

[Merle-Teissier] M. Merle, B. Teissier Conditions d'adjonction, d'après DuVal in Séminaire sur les Singularités des Surfaces Lecture Notes in Mathematics, 777. Springer, Berlin, 1980. 229-245

[Milnor-book] J. Milnor, Singular points of complex hypersurfaces, Annals of Math. Studies 61, Princeton University Press 1968.

[Morales1984] M. Morales, Polyèdre de Newton et genre gémérique d'une singularitéintersection complète. Bull. Soc. Math. France 112 (1984), no. $3,325-341$

[Morales1985] M. Morales, Fonctions de Hilbert, genre géométrique d’une singularité quasi homogène Cohen-Macaulay. C. R. Acad. Sci. Paris Sér. I Math. 301 (1985), no. 14, 699-702.

[Némethi98] A. Némethi, Dedekind sums and the signature of $f(x, y)+z^{N}$. Selecta Math. (N.S.) 4 (1998), no. 2, 361-376.

[Némethi99] A. Némethi, Dedekind sums and the signature of $f(x, y)+z^{N}$,II. Selecta Math. (N.S.) 5 (1999), $161-179$.

[Oka.1990] M. Oka, Principal zeta-function of nondegenerate complete intersection singularity. J. Fac. Sci. Univ. Tokyo Sect. IA Math. 37 (1990), no. 1, 11-32

[Oka] M. Oka, Non-degenerate complete intersection singularity. Actualités Mathématiques. [Current Mathematical Topics] Hermann, Paris, 1997. viii+309 pp

[Saito1981] M. Saito, On the exponents and the geometric genus of an isolated hypersurface singularity. Singularities, Part 2 (Arcata, Calif., 1981), 465-472, Proc. Sympos. Pure Math., 40, Amer. Math. Soc., Providence, RI, 1983.

[Seade-book] J. Seade, On the Topology of Isolated Singularities in Analytic Spaces. Progress in Mathematics 241, Birkhäuser 2006.

[Teissier1978] B. Teissier, On a Minkowski-type inequality for multiplicities. II. C. P. Ramanujam a tribute, pp. 347-361, Tata Inst. Fund. Res. Studies in Math., 8, Springer, Berlin-New York, 1978. 
[Teissier2004] B. Teissier, Monomial ideals, binomial ideals, polynomial ideals. Trends in commutative algebra, 211-246, Math. Sci. Res. Inst. Publ., 51, Cambridge Univ. Press, Cambridge, 2004.

[Tomari1993] M. Tomari, The inequality $8 p_{g}<\mu$ for hypersurface two-dimensional isolated double points. Math. Nachr. 164 (1993), $37-48$.

[Wahl1981] J. Wahl, Smoothings of normal surface singularities, Topology 20 (1981), 219-246.

[Xu-Yau1993] Y.-J. Xu, S.S.-T. Yau, Durfee conjecture and coordinate free characterization of homogeneous singularities. J. Differential Geom. 37 (1993), no. 2, 375-396.

[Yau-Zhang2006] St.-T.Yau, L.Zhang, An upper estimate of integral points in real simplices with an application to singularity theory. Math. Res. Lett. 13 (2006), no. 5-6, 911-921.

Department of Mathematics, Ben Gurion University of the Negev, P.O.B. 653, Be’er Sheva 84105, Israel, Dmitry.kerner@gmail.com, Rényi Institute of Mathematics, Budapest, Reáltanoda u. 13-15, 1053, Hungary, nemethi@Renyi.hu 